\title{
Botulinum toxin in facial plastic surgery
}

\author{
Nicole Favre, David Sherris
}

Department of Otolaryngology, Jacobs School of Medicine and Biomedical Sciences, State University of New York at Buffalo, Buffalo, NY 14209, USA.

Correspondence to: Dr. David Sherris, Department of Otolaryngology, Jacobs School of Medicine and Biomedical Sciences, State University of New York at Buffalo, 1237 Delaware Avenue, Buffalo, NY 14209, USA. E-mail: dsherris@buffalo.edu

How to cite this article: Favre N, Sherris D. Botulinum toxin in facial plastic surgery. Plast Aesthet Res 2020;7:71. http://dx.doi.org/10.20517/2347-9264.2020.149

Received: 8 Jul 2020 First Decision: 24 Aug 2020 Revised: 7 Sep 2020 Accepted: 2 Nov 2020 Published: 5 Dec 2020

Academic Editor: James E. Zins Copy Editor: Cai-Hong Wang Production Editor: Jing Yu

\begin{abstract}
Since the U.S. Food and Drug Administration approved botulinum toxin (BoNT) type A in 2002 for glabellar rhytids, BoNT has been used successfully for many clinical indications in facial plastic surgery. The current usage of BoNT as a non-invasive procedure for rhytids of the aging face include but are not limited to rhytids of the forehead, glabella, lateral orbit, nasal sidewall, upper lip, vertical perioral rhytids, melomental fold, and chin. In addition to facial rhytids, BoNT has been shown to be effective for a variety of other clinical indications in facial plastic surgery, including masseter hypertrophy, facial paralysis, brow ptosis, and wound healing. This article will review the pharmacology and mechanism of action of BoNT. In addition, the suggested dosage and instruction for injection for facial rhytids will be discussed along with BoNT usage for clinical indications other than rhytids.
\end{abstract}

Keywords: Botulinum toxin, botox, neurotoxin

\section{INTRODUCTION}

The first published report of botulinum toxin (BoNT) for aesthetic use in 1989 was just the beginning of the expansion of BoNT as one of the most common non-invasive techniques in the field of facial plastic surgery ${ }^{[1]}$. Shortly after this report, the safety and efficacy of BoNT-A to treat glabellar frown lines was demonstrated in study with injection into 18 patients by Carruthers and Carruthers ${ }^{[2]}$. Together with other trials, the U.S. Food and Drug Administration (FDA) approved the use of BoNT-A for glabellar rhytids in 2002. Although BoNT-A is currently approved by the FDA for the aesthetic treatment of various facial rhytids, there continues to be an increasing number of off-label aesthetic usages of $\mathrm{BoNT}^{[3]}$. According to

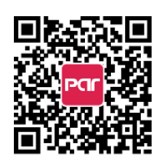


the American Society of Plastic Surgeons, in the United States specifically, the estimated BoNT-A injectable procedures performed in 2019 were approximately 7,697,798. This BoNT-A number is of anatomic sites injected and based on data from the 682 physicians included ${ }^{[4]}$. Because the face is the central aspect of a person's identity and a visible marker of one's age ${ }^{[5]}$, it is important to review the current non-invasive procedures used in aging face treatment. This article reviews the use of BoNT in the treatment of facial rhytids as well as other uses of BoNT in non-invasive procedures related to facial plastic surgery.

\section{MECHANISM OF ACTION}

BoNT is produced by Clostridium botulinum, a spore-forming obligate anaerobe that resides in the soil. BoNT is a simple dichain polypeptide that consists of a $100-\mathrm{kd}$ heavy chain joined by a single disulfide bond to a 50-kd light chain. BoNT acts by blocking the fusion of soluble N-ethylmaleimide sensitive factor attachment protein receptor (SNARE) proteins at the neuromuscular junction, resulting in the blocking of acetylcholine release and temporary flaccid paralysis ${ }^{[6]}$. The SNARE proteins targeted by different BoNTs vary. Although there are seven distinct types of BoNT, there are currently only two available for commercial usage, type A and type B. BoNT type A (BoNT-A) cleaves synaptosomal - associated protein (SNAP-25), whereas BoNT type B cleaves synaptobrevin or vesicle-associated membrane protein ${ }^{[7]}$. Although BoNT causes irreversible neuromuscular blockade as shown in both disease and therapy, muscle function does recover through the formation of axonal sprouts and new motor end plates, as well as the full recovery of the neuromuscular junction ${ }^{[3]}$.

There are currently three leading BoNT-A products available in the United States: OnabotulinumtoxinA (ONA; Botox), AbobotulinumtoxinA (ABO; Dysport), and IncobotulinumtoxinA (INCO; Xeomin). Although the conversation factors between the two formulations are not yet clearly stated, it is suggested in multiple studies that an ONA:ABO conversion ratio of exactly $1: 2$ is appropriate ${ }^{[8]}$. In a study by Hexsel et al. ${ }^{[9]}$, a conversion ratio of 1:2.5 for ONA:ABO showed significantly different fields of effect, which points to the fact that the difference in diffusion effect is specifically dose dependent. INCO was shown to be as effective as ONA with a comparable frequency of adverse effects when a clinical conversion ratio of 1:1 was used.

\section{INSTRUCTIONS FOR INJECTION}

When performing injections of BoNT-A, it is important to understand the anatomy of the muscle as well as the toxin, dilution, and needle characteristic used for injection in order to achieve the desired clinical outcome and avoid any complications or adverse effects. Based on the senior author's experience, the effect of BoNT-A starts at 24-48 h after injection, peaking at 7-14 days, and lasting 3-6 months, with an average of 3-4 months. There are some possible adverse effects that should be discussed with the patient prior to injection, including but not limited to unexpected loss of strength or muscle weakness, injection site reactions or bleeding, and bruising. Patients are instructed to avoid anticoagulants or other blood thinning medications for two weeks in order to minimize the bleeding and bruising ${ }^{[10]}$. It is also important to consider the diffusion of the neurotoxin when choosing which neurotoxin to use in each anatomic location. Some anatomic locations require a larger field of effect for optimal treatment, while this same large field of effect may produce an undesirable outcome for other anatomic locations ${ }^{[9]}$. Based on the prescribing information for each of these products, the 1:1 conversion ratio of ONA:INCO and the 1:2.5 conversion ratio of ONA:ABO are used in the tables below which summarize the injection dosage of facial rhytids [Tables 1-3].

\section{BONT TREATMENT FOR RHYTIDS OF THE FACE}

\section{Upper face}

Forehead

When treating the forehead to minimize rhytids, the main muscle of target is the frontalis muscle which attaches proximally to the galea aponeurotica and distally to the skin around the eyebrows and the nose. 
Table 1. Upper face rhytid dosage and injection instructions

\begin{tabular}{llll}
\hline Muscle & \multicolumn{1}{c}{ Onabotulinum } & \multicolumn{1}{c}{ Incobotulinum } & Abobotulinum \\
\hline Frontalis & 2-4 units over 4-6 injection sites & 2-4 units over 4-6 injection sites & 5-10 units over 4-6 injection sites \\
Glabellar Complex & 15-20 units over 5 injection sites & 15-20 units over 5 injection sites & 37.5-50 units over 5 injection sites \\
Lateral Orbit & 2-4 units over 3 injection sites & 2-4 units over 3 injection sites & 5-10 units over 3 injection sites \\
& bilaterally & bilaterally & bilaterally \\
\hline
\end{tabular}

Table 2. Midface rhytid dosage and injection instructions

\begin{tabular}{|c|c|c|c|}
\hline Muscle & Onabotulinum & Incobotulinum & Abobotulinum \\
\hline Nasalis & $\begin{array}{l}\text { 2-4 units bilaterally or one } \\
\text { central injection }\end{array}$ & $\begin{array}{l}\text { 2-4 units bilaterally or one } \\
\text { central injection }\end{array}$ & $\begin{array}{l}\text { 5-10 units bilaterally or one } \\
\text { central injection }\end{array}$ \\
\hline $\begin{array}{l}\text { Upper Lip (i.e., levator labii superioris } \\
\text { alaeque nasi, levator labii superioris, } \\
\text { zygomaticus minor) }\end{array}$ & $\begin{array}{l}\text { 4-6 units over } 2-4 \text { injection } \\
\text { sites }\end{array}$ & $\begin{array}{l}\text { 4-6 units over } 2-4 \text { injection } \\
\text { sites }\end{array}$ & $\begin{array}{l}10-15 \text { units over } 2-4 \text { injection } \\
\text { sites }\end{array}$ \\
\hline Orbicularis Oris & $\begin{array}{l}\text { 3-7 units over } 3 \text { injection } \\
\text { sites }\end{array}$ & $\begin{array}{l}\text { 3-7 units over } 3 \text { injection } \\
\text { sites }\end{array}$ & $\begin{array}{l}\text { 7.5-17.5 units over } 3 \text { injection } \\
\text { sites }\end{array}$ \\
\hline
\end{tabular}

Table 3. Lower face rhytid dosage and injection instructions

\begin{tabular}{llll}
\hline Muscle & \multicolumn{1}{c}{ Onabotulinum } & \multicolumn{1}{c}{ Incobotulinum } & Abobotulinum \\
\hline Depressor Anguli Oris & $2-3$ units into each muscle belly & $2-3$ units into each muscle belly & $5-7.5$ units into each muscle belly \\
Mentalis & 2 units into each muscle belly or 5 & 2 units into each muscle belly or 5 & 5 units into each muscle belly or 15 \\
& units into one midline injection & units into one midline injection & units into one midline injection \\
\hline
\end{tabular}

The main function of the frontalis muscle is to raise the eyebrows, and in doing so, it causes wrinkling of the forehead. In treating these rhytids caused by contraction of the frontalis muscle, it is important to differentiate between static and dynamic lines. Dynamic lines are a result of hyperfunctional muscle contraction, like that seen by the frontalis muscle when lifting the eyebrows, while static lines are unchanged with muscle movement and are more commonly treated with dermal fillers ${ }^{[11]}$.

Because the frontalis muscle is the only active muscle in this region, it is suggested that weakening of the muscle, instead of complete denervation, is preferred in order to avoid brow ptosis. The forehead furrows caused by the frontalis muscle respond favorably to either subcutaneous or intramuscular injection ${ }^{[10]}$ [Figure 1].

\section{Glabella}

The glabellar complex, a medial brow depressor, consists of muscles including procerus, corrugator supercilii, and depressor supercilii. The two main depressors are the corrugator supercilii which originates from the superciliary arch of the frontal bone and inserts into the skin of the eyebrow, and the procerus which originates from the fascia of the lower nasal bone and inserts on the skin between the eyebrows. Overall, the glabellar complex requires one injection site into the body of the procerus and 1 to 2 injection sites on each side for the corrugator supercilii muscle [Figure 2]. The more complex treatment of the corrugator supercilii involves a deeper injection at the medial portion to capture the depressor supercilii and a more superficial injection laterally when the muscle approaches the dermis ${ }^{[10]}$. The treatment of glabellar rhytids was the first approved aesthetic use of BoNT-A, and since then, several follow up studies have shown effective treatment for these specific rhytids ${ }^{[12]}$.

A major side effect from improper injection of the glabellar complex, as well as all other neurotoxin injections of the upper face, is eyelid ptosis. For the glabellar complex specifically, it is necessary to inject above the orbital rim to prevent diffusion of the neurotoxin to the levator palpebrae superioris muscle, which would result in eyelid ptosis ${ }^{[13]}$. Ptosis can occur up to 2 weeks after injection. If eyelid ptosis from migration to the levator palpebrae superioris occurs, it can be treated with Apraclonidine (Lopidine) eye drops, which is an alpha-2-adrenergic agonist that causes the superior tarsal muscle to contract and elevate the upper eyelid ${ }^{[14]}$. 


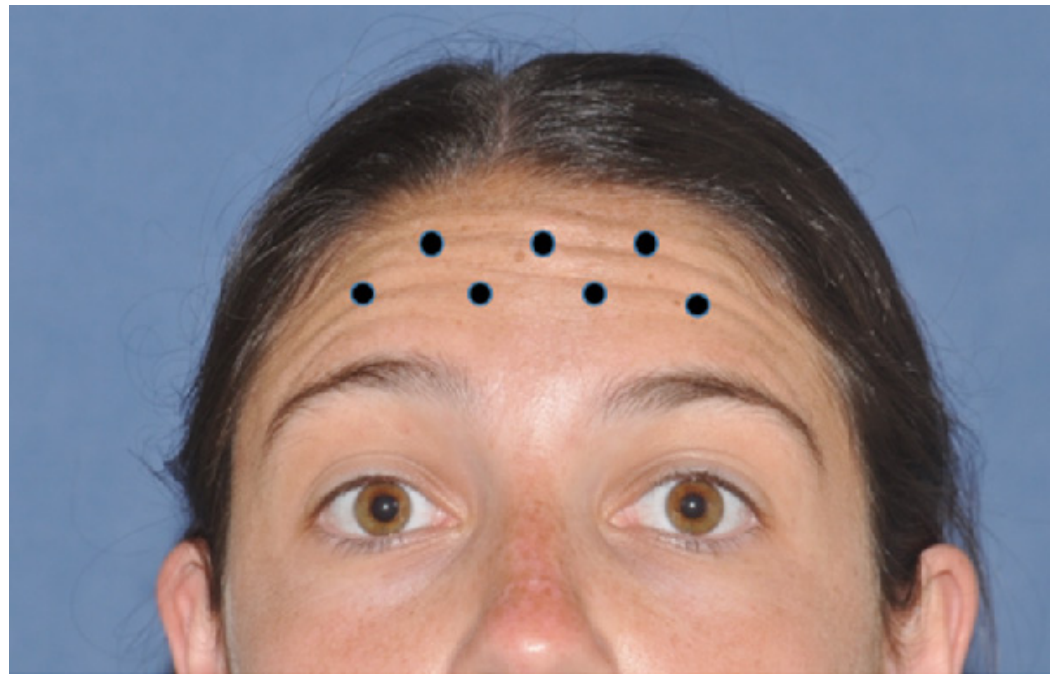

Figure 1. Injection sites for forehead rhytids into the frontalis muscle

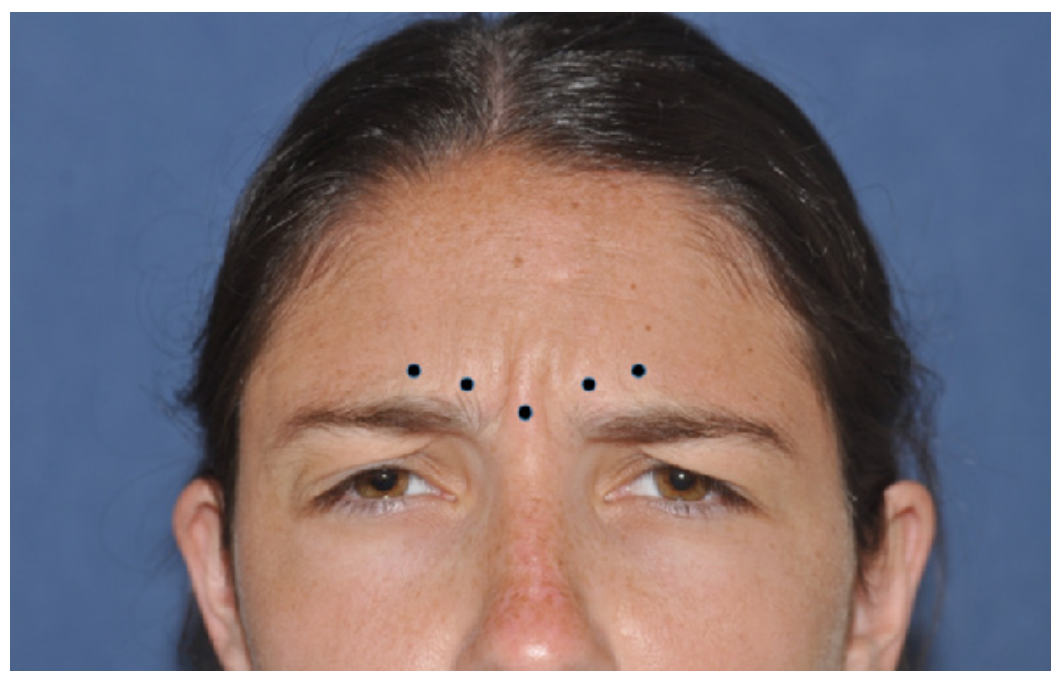

Figure 2. Injection sites for glabellar rhytids into the procerus and corrugator supercilii muscle

\section{Lateral orbit}

The main muscle of the lateral orbit is the orbicularis oculi muscle, the muscle involved in closure of the eye and depression of the eyebrow. This muscle is divided into three parts: the orbital, the preseptal, and the pretarsal portions. The muscle extends beyond the orbital rim and creates lateral rhytids known as crow's feet. Treatment of these rhytids can be done with one or three injections on either side [Figure 3]. In a two-center randomized study by Fabi et al.$^{[15]}$, no statistically significant difference was seen in crow's feet treated with one injection per side $v s$. crow's feet treated with three injections per side. In addition, there was no difference in adverse events based on injection number. With the use of three injections per side, the middle injection should be in line with the lateral canthus and the remaining should be 8 to $10 \mathrm{~mm}$ away. It is critical to inject at least $1 \mathrm{~cm}$ lateral to the lateral orbital rim in order to avoid complications, such as diplopia or strabismus, from diffusion into the extraocular muscles resulting in the paralysis of the lateral rectus muscle ${ }^{[13]}$. In addition to the crow's feet rhytids, hypertrophy of the pretarsal portion of the orbicularis oculi can cause what is known as the "jelly roll" appearance around the lower eyelid. When a person is smiling, the contraction of the pretarsal portion diminishes the side of the palpebral aperture 


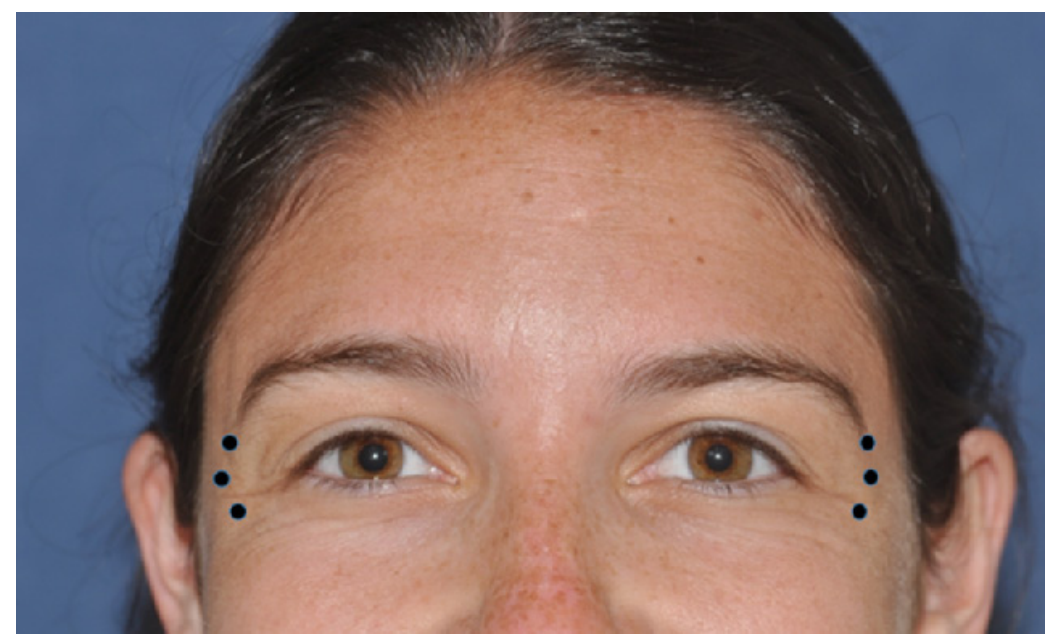

Figure 3. Injection sites for lateral orbit (crow's feet) rhytids into the orbicularis oculi muscle

which causes these "jelly roll" lines. It is recommended that 2 units of ONA be injected into the pretarsal portion of the orbicularis oculi to counteract the contraction causing the diminished palpebral aperture ${ }^{[16]}$.

One specific consideration regarding patient satisfaction that should be considered when using BoNT for crow's feet rhytids is the interaction between the dynamic lines, treated by the neurotoxin, and the static lines in this region. The junction of these static and dynamic lines creates rhytids that the patient may not be satisfied with after neurotoxin injection. These static rhytids should then be treated with fillers, such as hyaluronic acid injections, which increase the volume of the skin in this area and remove the lines created by the junction of static and dynamic rhytids ${ }^{[10]}$.

\section{Midface}

Nasal sidewalls

Rhytids in the region of the upper nose, known as "bunny lines," are a result of contraction of the upper, transverse portion of the nasalis muscle. Recommended injection for treating these "bunny lines" is 2 to 4 units of ONA directly into the nasalis muscle on each side of the nose or one central injection at the midline [Figure 4]. The location of injection is of extreme importance in this region. Injections placed too low on the nose, below the nasofacial groove, can cause relaxation of the levator labii superioris alaeque nasi and levator anguli oris, resulting in both lip ptosis and smile asymmetry. On the other hand, injections placed too high could cause medial rectus paresis and epiphora ${ }^{[17]}$.

One other usage of BoNT in the nose region is to treat the effects of nasal flare, which is when a patient repeatedly involuntarily dilates their nostrils in social situations. This dilation makes the nasal septum visible and causes an undesired appearance that results in lack of self-esteem and confidence in social settings. It is recommended that injection of 5 to 10 units of ONA into the portion of the nasalis muscle over the nasal ala will help to inhibit this involuntary nasal flare ${ }^{[16]}$.

\section{Upper lip}

A person's smile is thought to be one of the most meaningful facial expressions in today's society; therefore, it is important to ensure patient satisfaction of the aesthetic appearance of their smile. One of the most desired changes to one's smile is removal of excessive gingival exposure, more commonly known as "gummy smile." The "gummy smile" is defined as greater than or equal to $2 \mathrm{~mm}$ of gingival exposure while smiling ${ }^{[18]}$. Gingival exposure may need surgical intervention based on the specific cause, such as from 


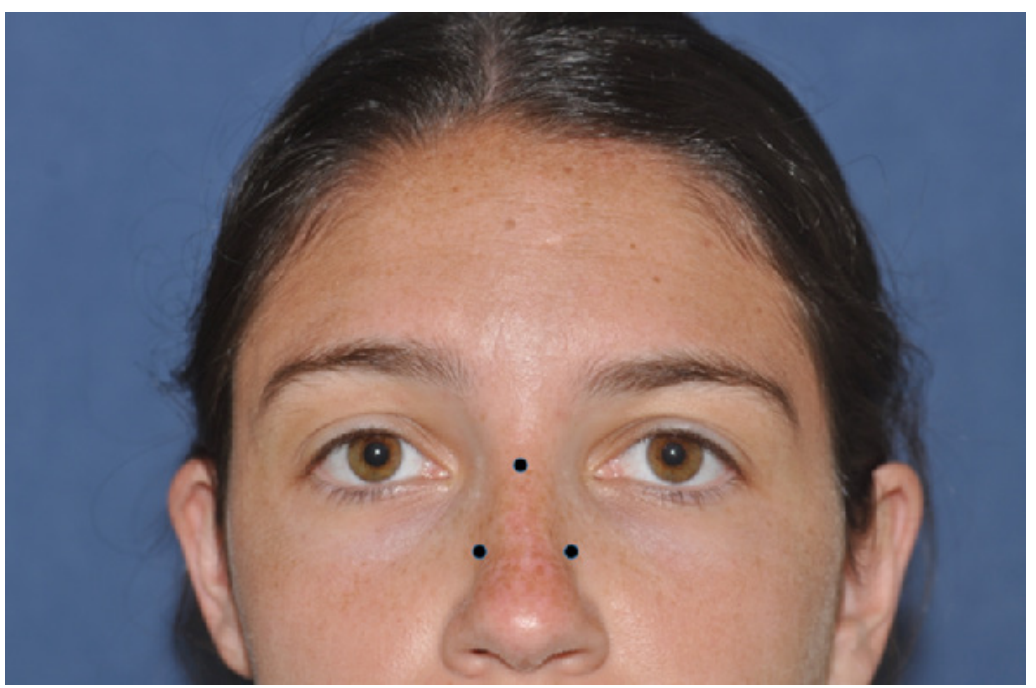

Figure 4. Injection sites for nasal sidewall rhytids into the nasalis muscle

delayed dental eruption or vertical maxillary excess; however, gingival exposure due to muscle hypertrophy can be treated with BoNT. The "gummy smile" in this case is caused by overactivation of the muscles that elevate the central lip, including the levator labii superioris, levator labii superioris alaeque nasi, and the zygomaticus minor. It is recommended to inject 2 to 4 sites of the lip elevator muscles with 4 to 6 units of ONA [Figure 5]; studies show that a higher dosage is necessary for more extreme gingival exposure ${ }^{[19]}$. While an asymmetric smile can be corrected with the use of BoNT-A on the levator labii superioris alaeque nasi, it is important to be cautious of dosage and location of injection so as to not create an asymmetric smile with the paralysis of the depressor labii inferioris muscle.

\section{Vertical perioral rhytids}

Vertical perioral rhytids are caused by the activation of the orbicularis oris muscle, which encircles the mouth, inserting directly on the lips and controlling movements of the mouth and the lips. It is recommended that 3 to 7 units of ONA are injected over three sites in the upper lip. Because it is more difficult to assess patient response with the orbicularis oris muscle than muscles of the forehead and upper face, careful consideration of dosage is important to avoid complete paralysis of the muscle, resulting in the patient's inability to purse their lips, drink from a straw, or phonate clearly ${ }^{[16]}$. It is advised to start with lower doses so the patient has time to determine their satisfaction of the treatment response.

\section{Lower face}

\section{Melomental folds}

The activity of the depressor anguli oris muscle on either side of the mouth is responsible for depressing the corner of the mouth. Hyperactivity of these muscles can result in the appearance of being sad, tired or angry in some patients. Treatment with BoNT-A can be used to relax these hyperactive muscles and achieve desired facial appearance.

Injection of BoNT-A into the depressor anguli oris muscle is difficult because of its fan shape with its medial portion overlapping with the depressor labii inferioris muscle and its lateral portion located in close proximity to the risorius, zygomaticus major and platysma muscles. Choi et al ${ }^{[20]}$ showed that the best location for injection to avoid unwanted side effects with diffusion of the neurotoxin to other muscle groups is the inferior portion of the muscle located at the mandible, enclosed in the fan shaped area [Figure 6]. 


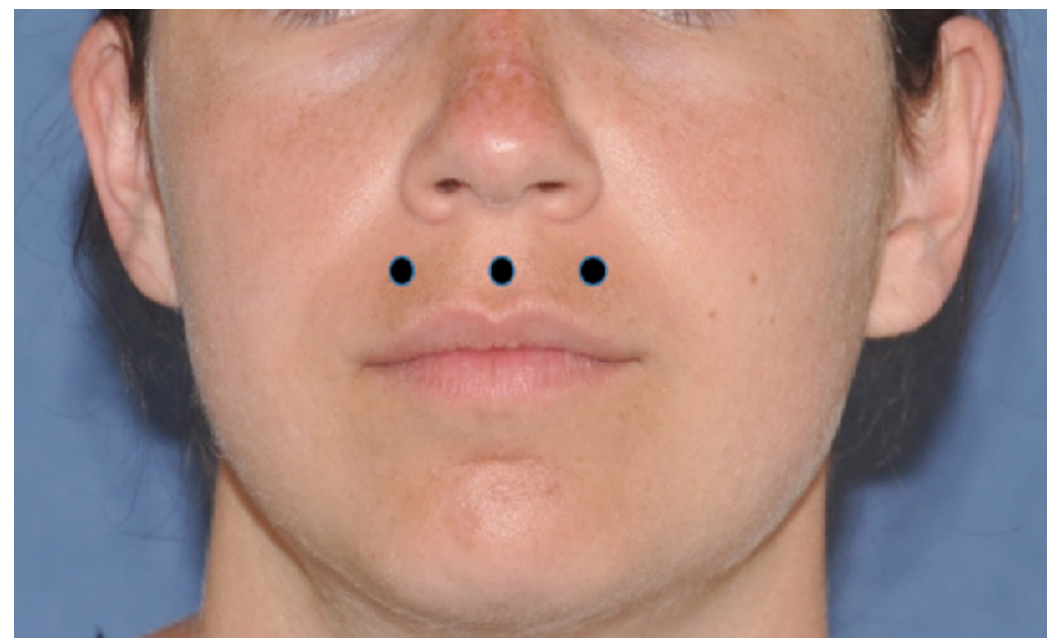

Figure 5. Injection sites for upper lip rhytids into the lip elevator muscles

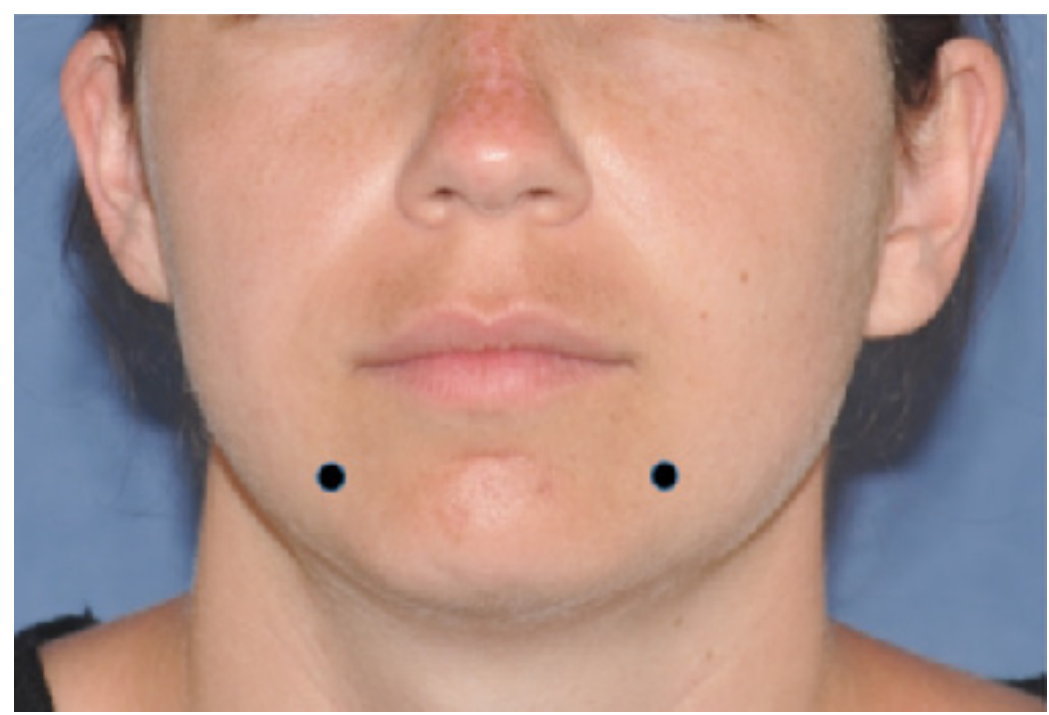

Figure 6. Injection sites for melomental folds into the depressor anguli oris muscle

\section{Chin}

One of the most common areas of the face frequently injected with BoNT for aesthetic reasons is the chin, with the main muscle of injection being the mentalis muscle. The mentalis has two muscle bellies that originate from the mentum and insert onto the chin soft tissue under the lip. Its function is to elevate and protrude the lower lip while wrinkling the skin of the chin, often producing the emotions of doubt or "pouting" ${ }^{[13]}$. Injections to improve the wrinkling of the chin and aesthetic appearance of this area can be performed with a single injection in the midline of the chin with 5 units of ONA or bilaterally in each of the muscle bellies with 2 units of ONA per injection ${ }^{[3]}$ [Figure 7]. Patients are often satisfied with the results of decreased wrinkling in the mental area after injection.

\section{OTHER USES OF BONT}

\section{Masseteric hypertrophy}

The masseter muscle originates from the inferior border of the zygomatic arch and inserts onto the angle and ramus of the mandible, functioning to elevate and protract the mandible to close the jaw. Hypertrophy 


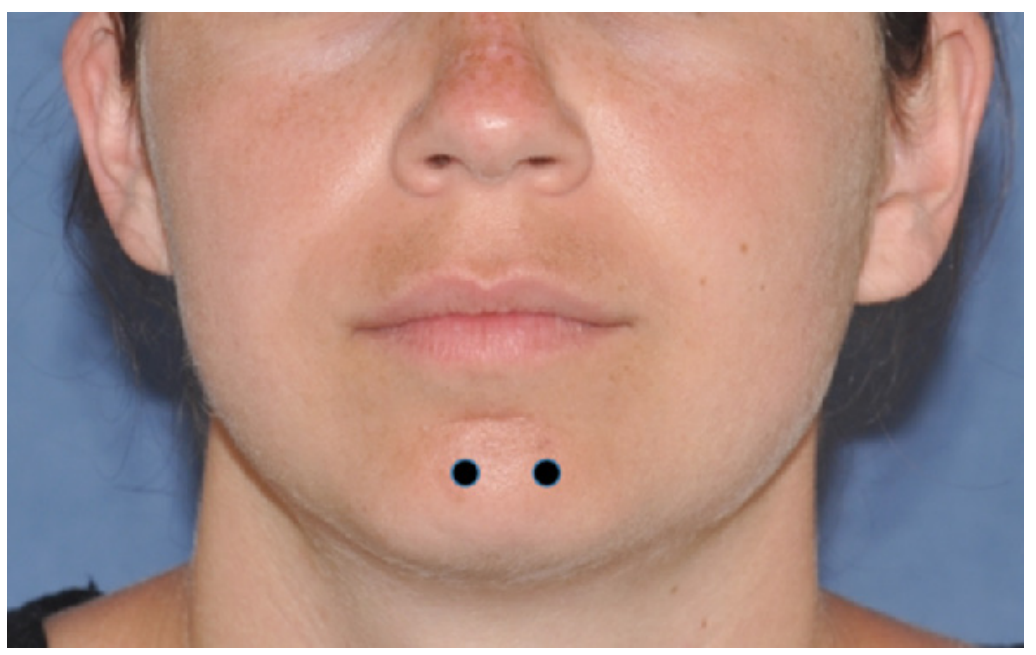

Figure 7. Injection sites for chin rhytids into the mentalis muscle

of this muscle can cause the lower face to look square, which is often not a desired appearance for female patients. While the goal of treatment for the facial rhytids described above was to induce muscle paralysis, the goal for BoNT-A injection of the masseter muscle is to soften the square appearance of the face by reducing masseter muscle mass, ultimately creating a more desired aesthetic appearance ${ }^{[3]}$. Dosage of BoNT-A for injection is generally around 15 to 20 units of ONA, but the specific dosage depends on the degree of hypertrophy. The effects of this injection can last six to nine months, but, as with other BoNT-A injections, patients often follow up multiple weeks after injection to determine the need for more injections. Factors such as bruxism and clenching habits can increase masseter muscle thickening and require more frequent BoNT-A injections ${ }^{[21]}$. In addition to BoNT-A's aesthetic effects on the masseter muscle, patients with temporomandibular joint dysfunction (TMJ) symptoms that have masseter hypertrophy report a relief of their TMJ symptoms with BoNT-A injections ${ }^{[10]}$.

The injector must be careful to avoid injecting the risorius muscle, which can result in the undesired side effect of an asymmetric smile. It is important to inject inferiorly and laterally along the masseter muscle to avoid this negative effect. In addition, overdosage of BoNT-A could result in inability to chew ${ }^{[3]}$.

\section{Platysmal bands}

In addition to the upper, middle, and lower face, the neck is another region of the body where BoNT treatment as a non-invasive procedure can be beneficial. The platysma muscle is the main muscle of the neck. Unlike most muscles in the body, the platysma is located just below the epidermis and dermis in the superficial layer of fascia. This location in relation to the skin is one of the reasons why this muscle is an important muscle in aging. The platysma is a muscle of facial expression with many roles, including depression of the mandible, depression of the corners of the mouth in frowning, and tensing of the skin of the neck. Treatment with BoNT-A can counteract this downward pull in order to soften the appearance of the neck and provide jawline definition ${ }^{[10]}$.

Since the platysma muscle is located in the superficial layer of fascia, it is important to ensure that the BoNT-A does not spread to the deeper muscles when injected. To prevent this spread, it is recommended that the patient contract their platysma muscle in order for the physician to grasp the muscle with his/her fingers and inject the neurotoxin at a superficial level. Injections not performed this way could result in serious complications such as dysphagia ${ }^{[22]}$. 


\section{Facial paralysis}

Facial paralysis is a cause for patient concern both aesthetically and functionally. Aesthetically, patients develop facial asymmetry from this flaccid paralysis, while functionally, patients can develop difficulty with speaking, eating, breathing, and ocular problems ${ }^{[3]}$. In addition to this flaccid paralysis, patients can experience secondary effects of facial palsy healing including hyperkinesis, which consists of static and dynamic asymmetry of the face due to hypertonia, and synkinesis, which is involuntary movement of certain groups of mimic muscles ${ }^{[23]}$. These problems not only affect the patient's ability to function in their daily activities but can also result in psychological and social problems due to their inability to clearly communicate with others.

Facial paralysis has been treated with minor operations such as brow lifts, medial canthoplasty, and orbicularis myectomy, as well as major procedures such as facial-hypoglossal anastomosis, vascular free muscle grafts, and cross-facial surgery ${ }^{[23]}$. BoNT, therefore, can be used as a less invasive method to treat facial nerve paralysis by blocking presynaptic release of acetylcholine resulting in temporary denervation. There is no standard of dosing or location for BoNT-A injection for facial paralysis; treatment is determined based on the clinical evaluation of the individual patient.

\section{Brow ptosis}

Brow ptosis is often related to incorrect injection of BoNT in an unwanted area, leading to muscle weakness and the aesthetic appearance of an eye brow droop ${ }^{[24]}$. However, BoNT, used correctly, can actually help to relieve brow ptosis and even out the eyebrows aesthetically. Injection of 2 units of ONA at 2 to $3 \mathrm{~mm}$ below the lateral brow and another injection of 2 units of ONA into the corrugator at the medial brow results in elevation of the brow because of the reduced action of the depressor muscles ${ }^{[24}$ [Figure 8]. The other alternative treatment, if that eyebrow is not able to be raised, is to lower the opposite eyebrow by injecting BoNT in the brow elevators above the eyebrow. This works to achieve symmetry of the eyebrows, but should be done only if elevating the affected eyebrow is not possible ${ }^{[25]}$.

\section{Process of healing}

BoNT has been shown in many studies to improve appearance of scar formation with a normal healing wound through the mechanism of relieving the tension of the skin at the scar region by blocking muscle contraction in that area ${ }^{[26]}$. In addition to effects on normal wound healing, there have been interesting advancements in the usage of BoNT-A for the treatment of pathological scarring, including hypertrophic scars and keloids. Pathologic scars can form from defects in any of the three overlapping processes of healing: the initial inflammatory phase, the proliferative phase with formation of scar tissue, and finally, the tissue remodeling phase ${ }^{[27]}$. Both hypertrophic scars and keloids are firm, erythematous plaques or nodules, but a main difference is that hypertrophic scars are confined to the margin of the wound, while keloids often grow beyond the boundaries of the wound ${ }^{[28]}$.

The exact mechanism of BoNT treatment for pathologic scars is still unclear, but multiple theories have been reviewed in the current literature. One of the important factors in pathologic scar formation where BoNT-A could be of benefit is the tension on the wound caused by the contraction of muscles on the wound edges, which is the same mechanism found in normal healing and scar formation ${ }^{[29]}$. Another mechanism of pathologic scarring where BoNT-A treatment could help is the increased collagen synthesis and increased metabolic activity that results due to local inflammation in the pathologic scarring processes. The injection of BoNT-A is thought to minimize the tension on the wound, allowing the maturation of collagen ${ }^{[30]}$. A final theory is fibroblast hyperproliferation in the formation of both hypertrophic scars and keloids. One study by Austin et al. ${ }^{[31]}$ showed that BoNT-A can directly decrease fibroblastic activity through alteration of apoptotic, migratory, and fibrotic pathways in scar formation to improve the pathologic scar appearance Although the exact mechanism for the treatment of hypertrophic scars 


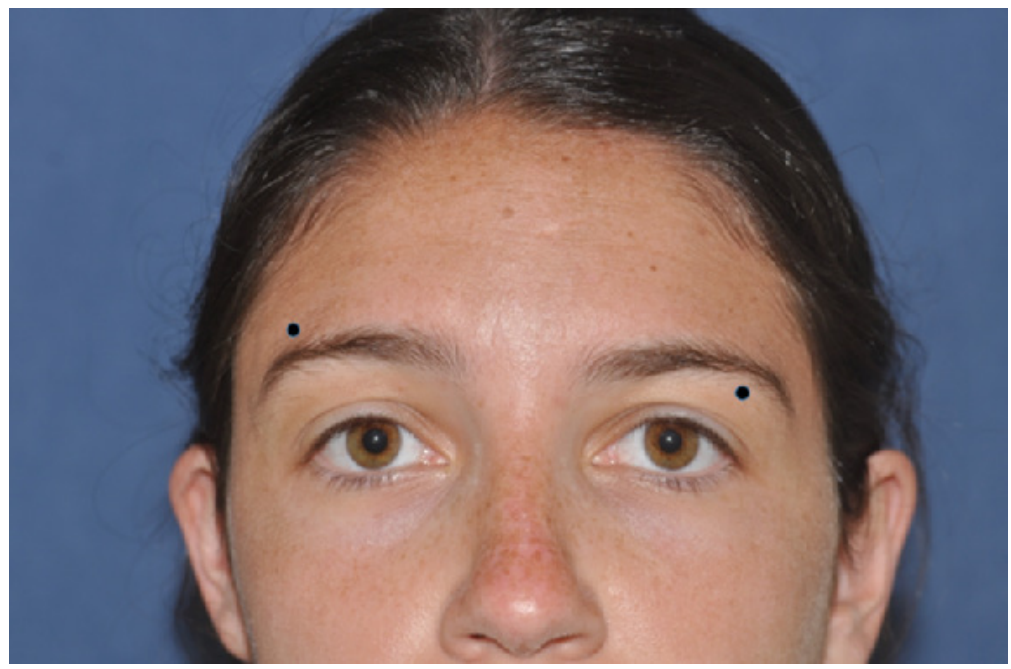

Figure 8. Injection sites above the elevated eyebrow or below the depressed eyebrow in brow asymmetry from brow ptosis

and keloids by BoNT is currently not completely understood, several studies have pointed to a potential expansion the off-label use of BoNT-A for pathologic scarring.

\section{COMPLICATIONS}

Although there are specific complications and contraindications with usage of BoNT for specific muscle groups that were discussed above, there are general complications to be aware of regardless of where the injection is located. Locally, the complications are most commonly related to the injection site. These complications include development of pain, erythema, ecchymosis, or hematoma at the site of injection. It is often helpful to use local anesthetics and inject the neurotoxin at a slower rate to avoid these injection site complications. It is important to inform your patients that ecchymosis is common and can take up to two weeks to resolve ${ }^{[32]}$. As discussed in detail above, another common side effect is diffusion of the neurotoxin to adjacent muscle groups resulting in paralysis of those muscle groups and unwanted aesthetic as well as functional side effects. Care must be taken to ensure the toxin is injected directly into the desired muscle $^{[33]}$.

Some less common complications of BoNT injection include development of a mild and self-resolving headache with facial injections, paresthesia in the area of the injection site caused by local nerve trauma, and vasovagal episodes associated with anxiety during the injection procedure ${ }^{[32]}$. It is important to be aware of these side effects in order to avoid further complications. Finally, two extremely rare complications to note are the formation of antibodies against the BoNT, which would make the treatment ineffective, and the possible immediate allergic reactions to the toxin, which would present with itching, swelling, and possible anaphylaxis ${ }^{[34]}$. For the formation of antibodies, medications that inhibit neuromuscular signaling can be used to increase the toxin's effect by offsetting the antibody reaction. In the case of an allergic reaction, standard allergy protocol is recommended, but diphenhydramine is not recommended due to is anticholinergic effects.

\section{CONCLUSION}

BoNT has been proven to be safe and efficacious for many clinical indications in the field of facial plastic surgery. The use of BoNT for rhytids of the face has become one of the most common non-invasive procedures in facial plastic surgery with an extremely high patient satisfaction rate. In addition to the FDA approved usage of BoNT for rhytids, off-label uses, such as those described above, are becoming 
increasingly common. Although there are some possible complications to be aware of, most patients do extremely well with this treatment. New uses for BoNT in this field are constantly arising and are worth exploring further.

\section{DECLARATIONS}

\section{Authors' contributions}

Conception and design of the work: Sherris D

Analysis and interpretation of data for the work: Favre N, Sherris D

Drafting the work and revising it critically for important intellectual content: Favre N, Sherris D

Final approval of the version to be published: Favre N, Sherris D

\section{Availability of data and materials}

The data that support the findings of this study are available from the corresponding author, upon reasonable request.

\section{Financial support and sponsorship}

None.

\section{Conflicts of interest}

All authors declared that there are no conflicts of interest.

\section{Ethical approval and consent to participate}

Not applicable.

\section{Consent for publication}

I (Nicole Favre) consent to use my pictures.

\section{Copyright}

(c) The Author(s) 2020.

\section{REFERENCES}

1. Clark RP, Berris CE. Botulinum toxin: a treatment for facial asymmetry caused by facial nerve paralysis. Plast Reconstr Surg 1989;84:353-5.

2. Carruthers JD, Carruthers JA. Treatment of glabellar frown lines with C. botulinum-A exotoxin. J Dermatol Surg Oncol 1992;18:17-21.

3. Ong AA, Sherris DA. Neurotoxins. Facial Plast Surg 2019;35:230-8.

4. American Society of Plastic Surgeons News Release. Plastic Surgery Statistics Report. Available from: https://www.plasticsurgery.org/ news/plastic-surgery-statistics. [Last accessed on 10 Nov 2020]

5. Berwick S, Humble Á. Older women's negative psychological and physical experiences with injectable cosmetic treatments to the face. $J$ Women Aging 2017;29:51-62.

6. Huang W, Foster JA, Rogachefsky AS. Pharmacology of botulinum toxin. J Am Acad Dermatol 2000;43:249-59.

7. Jankovic J, Brin MF. Therapeutic uses of botulinum toxin. N Engl J Med 1991;324:1186-94.

8. Hexsel D, Brum C, do Prado DZ, et al. Field effect of two commercial preparations of botulinum toxin type A: a prospective, doubleblind, randomized clinical trial. J Am Acad Dermatol 2012;67:226-32.

9. Hexsel D, Soirefmann M, Porto MD, Siega C, Schilling-Souza J, Rodrigues TC. Fields of muscular and anhidrotic effects of 2 botulinum toxin-A commercial preparations: a prospective, double-blind, randomized, multicenter study. Dermatol Surg 2015;41 Suppl 1:S110-8.

10. Cohn JE, Greco TM. Advanced techniques for the use of Neurotoxins in non-surgical facial rejuvenation. Aesthetic Plast Surg 2020;44:1788-99.

11. Rohrich RJ, Ghavami A, Crosby MA. The role of hyaluronic acid fillers (Restylane) in facial cosmetic surgery: review and technical considerations. Plast Reconstr Surg 2007;120:41S-54.

12. Carruthers JD, Lowe NJ, Menter MA, Gibson J, Eadie N, Botox Glabellar Lines II Study Group. Double-blind, placebo-controlled study of the safety and efficacy of botulinum toxin type A for patients with glabellar lines. Plast Reconstr Surg 2003;112:1089-98.

13. Beer JI, Sieber DA, Scheuer JF 3rd, Greco TM. Three-dimensional facial anatomy: structure and function as it relates to injectable 
neuromodulators and soft tissue fillers. Plast Reconstr Surg Glob Open 2016;4:e1175.

14. Scheinfeld N. The use of apraclonidine eyedrops to treat ptosis after the administration of botulinum toxin to the upper face. Dermatol Online J 2005;11:9.

15. Fabi SG, Sundaram H, Guiha I, Goldman MP. A two-center, open-label, randomized, split-face study to assess the efficacy and safety of one versus three intradermal injection sites of abobotulinumtoxinA in the treatment of lateral periocular rhytides. J Drugs Dermatol 2013;12:932-7.

16. Carruthers J, Carruthers A. Aesthetic botulinum A toxin in the mid and lower face and neck. Dermatol Surg 2003;29:468-76.

17. Loos BM, Maas CS. Relevant anatomy for botulinum toxin facial rejuvenation. Facial Plast Surg Clin North Am 2003;11:439-43.

18. Suber JS, Dinh TP, Prince MD, Smith PD. OnabotulinumtoxinA for the treatment of a "gummy smile". Aesthet Surg J 2014;34:432-7.

19. Sucupira E, Abramovitz A. A simplified method for smile enhancement: botulinum toxin injection for gummy smile. Plast Reconstr Surg 2012;130:726-8.

20. Choi YJ, Kim JS, Gil YC, et al. Anatomical considerations regarding the location and boundary of the depressor anguli oris muscle with reference to botulinum toxin injection. Plast Reconstr Surg 2014;134:917-21.

21. Kim NH, Park RH, Park JB. Botulinum toxin type A for the treatment of hypertrophy of the masseter muscle. Plast Reconstr Surg 2010;125:1693-705.

22. Matarasso A, Matarasso SL. Botulinum A exotoxin for the management of platysma bands. Plast Reconstr Surg 2003;112:138S-40.

23. Filipo R, Spahiu I, Covelli E, Nicastri M, Bertoli GA. Botulinum toxin in the treatment of facial synkinesis and hyperkinesis. Laryngoscope 2012;122:266-70.

24. King M. Management of Ptosis. J Clin Aesthet Dermatol 2016;9:E1-4.

25. Olson JJ. Balanced botox chemodenervation of the upper face: symmetry in motion. Semin Plast Surg 2007;21:47-53.

26. Gassner HG, Brissett AE, Otley CC, et al. Botulinum toxin to improve facial wound healing: A prospective, blinded, placebo-controlled study. Mayo Clin Proc 2006;81:1023-8.

27. Li J, Chen J, Kirsner R. Pathophysiology of acute wound healing. Clin Dermatol 2007;25:9-18.

28. Ghazawi FM, Zargham R, Gilardino MS, Sasseville D, Jafarian F. Insights into the pathophysiology of hypertrophic scars and keloids: How do they differ? Adv Skin Wound Care 2018;31:582-95.

29. Kasyanju Carrero LM, Ma WW, Liu HF, Yin XF, Zhou BR. Botulinum toxin type A for the treatment and prevention of hypertrophic scars and keloids: updated review. J Cosmet Dermatol 2019;18:10-5.

30. Xiao Z, Qu G. Effects of botulinum toxin type a on collagen deposition in hypertrophic scars. Molecules 2012;17:2169-77.

31. Austin E, Koo E, Jagdeo J. The cellular response of keloids and hypertrophic scars to Botulinum toxin A: a comprehensive literature review. Dermatol Surg 2018;44:149-57.

32. Small R. Botulinum toxin injection for facial wrinkles. Am Fam Physician 2014;90:168-75.

33. Batniji RK, Falk AN. Update on botulinum toxin use in facial plastic and head and neck surgery. Curr Opin Otolaryngol Head Neck Surg 2004;12:317-22.

34. Naumann M, Carruthers A, Carruthers J, et al. Meta-analysis of neutralizing antibody conversion with onabotulinumtoxinA (BOTOX®) across multiple indications. Mov Disord 2010;25:2211-8. 